\title{
La Cistopexia modificada
}

\author{
Dres. : Isaías Arenas Buenahora y J esús Francisco Cadena
}

Clínica Santa Teresa - Bucaramanga

La mayor parte de lo que conocemos de la continencia urinaria, en la mujer normal, lo hemos aprendido de la incontinencia urinaria de esfuerzo (IUE) (T. Stamey).

\section{INTRODUCCION}

La incontinencia urinaria de esfuerzo, es la causa más común de pérdida de orina en forma involuntaria, y muchos investigadores han demostrado que hasta el $50 \%$ de las mujeres nulíparas acusa un cierto grado de pérdida de orina en forma involuntaria.

Así es como se define: pérdida de orina en forma involuntaria, a través de la uretra intacta, usualmente causada por un aumento de la presión intraabdominal, en una cantidad suficiente para ser socialmente inaceptable.

\section{OBJETIVOS}

La orina residual no debe estar presente y su ausencia debe comprobarse en el exámen cl ínico.

Algunas pacientes con vejigas atónicas, de grandes cantidades de orina residual, pueden presentarse con una historia similar a la de la incontinencia urinaria de esfuerzo, pero estas pacien- tes teóricamente no se benefician con la cirugía.

En la definición de incontinencia urinaria de esfuerzo, se presupone que no existe infección urinaria y cuando tal hecho está presente deben descartarse las diversas causas, se somete a las pacientes a tratamiento específico con el fin de obtener en lo posible la resolución del problema infección.

Sin embargo, en la serie estudiada, se consideró que el cistocele podría ser factor para producir o mantener la infección.

En el estudio de incontinencia urinaria pura, una vez que se ha demostrado que no existe orina residual, o infección urinaria, el exámen más útil y sencillo es la Uretrocistografía hecha en posición vertical, usando la cadena y la maniobra de Valsalva.

Jeffcoate y Roberts en 1952 y 1955 comprobaron que "la continencia podía ser preservada en mujeres con todos los grados de relajación pélvica aún con prolapsos uterovaginales, si la vejiga mantenía su base razonablemente plana en relación con la uretra, y un ángulo de 90 grados más o menos 30; o sea el ángulo uretro-vesical posterior". 
Basados en esto y en los estudios de Hutch en 1967, Tanacho (1974) y Stamey, que comprobaron que existen varios tipos de cirugía para corregir la incontinencia urinaria de esfuerzo, sin que ninguna de ellas obtenga el $100 \%$ de éxito y como se comprobó en Stanford en las experiencias de Stamey, las intervenciones que producen éxito "están siempre acompañadas de un desplazamiento hacia arriba y hacia adelante de la unión uretrovesical".

Las operaciones que fracasan, se caracterizan por falla en la movilización de este ángulo. Los éxitos posteriormente se convierten en fracasos invariablemente $y$ se asocian con regreso de la unión uretrovesical hacia abajo $\mathrm{y}$ hacia atrás, a la posición de incontinencia pre-operatoria.

Analizando los distintos tipos de intervención quirúrgica, se llegó a la conclusión de que solamente las intervenciones suprapúbicas son capaces de obtener una elevación de la unión uretrovesical hacia arriba y hacia adelante.

Que la intervención de MarshallMarchetti-Krantz, la intervención de Pereyra y su modificación, la de Stamey, ofrecen buenos resultados, pero presentan dificultades técnicas que elevan la morbilidad y llevan a complicaciones como las fístulas que habitualmente fracasan debido al temor del cirujano a tomar los puntos de suspensión con la cantidad de tejido suficiente y necesario para obtener una adecuada fijación.

Estas consideraciones nos llevaron a pensar en la necesidad de disponer de un campo quirúrgico que nos permitiera tener los fondos de sacos vaginales a nuestra entera disposición, de tal manera que los puntos de fijación se hicieran tomando la cantidad de tejido necesario y en la ubicación conveniente para obtener un soporte que llevara hacia adelante y hacia arriba la unión uretrovesical.
Para obtener esto era necesario practicar la histerectomia y obtener el paso de los puntos de fijación justo hacia la porción ósea pélvica anterior. Por esta razón se resolvió hacer tunelización extraperitoneal y el único sitio que permitía la fijación formando una verdadera faja de suspensión o "cincha" que sostuviese la unión uretrovesical hacia adelante y hacia arriba, era llevando estos puntos al ligamento de Cooper. Esto constituia una modificación a la técnica de Burch.

Al comunicar nuestra inquietud al ginecólogo, él vio el beneficio que podría derivarse para aquellas pacientes que no solamente padecían de incontinencia $y$ cistocele para las cuales una histerectomía podría pensarse ser excesiva cirugía; sino en aquellas pacientes con problemas ginecológicos asociados. De aquí nació nuestro trabajo que hoy a manera de informe preliminar, y con el único afán de comunicar una experiencia clínica, que creemos, una vez divulgada, suficientemente probada y posiblemente modificada, será un recurso más en el ya numeroso armamentario quirúrgico que busca corregir el problema tan frecuente del escape urinario involuntario en la mujer.

\section{MATERIAL Y METODOS}

Se ha practicado esta intervención a 68 pacientes procedentes de la clientela privada, del Instituto de Seguro Social, de la Caja Nacional de Previsión y algunas otras instituciones de cobertura médica social asistencial.

La primera intervención se practicó en marzo de 1981 y la última de las aqui reportadas en agosto de 1983.

En el cuadro No. 1 se aprecia que el $76.4 \%$ de las pacientes tienen más de 40 años. Este dato es muy importante para la consideración de la práctica de 
Cuadro No. 1

DISTRIBUCION ETAREA

No. de Casos

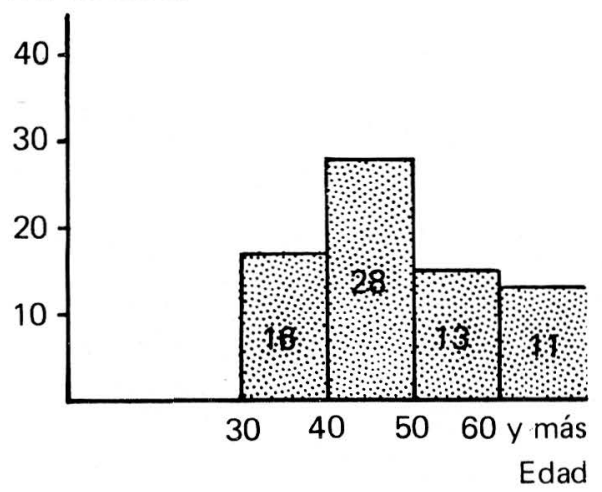

la histerectomía, ya que en nuestro medio se considera a la matriz como "órgano de satisfacción sexual" sin el cual el esposo no encontrará a su esposa "normal".

\section{Antecedentes}

34 pacientes o sea el $50 \%$ (cuadro No. 2) tenían cirugía previa que buscaba corregir en su gran parte o mayoría la incontinencia urinaria y el problema ginecológico.

22 pacientes, $32.35 \%$, se habían sometido a cirugía vaginal tipo Kelly-Ken. nedy o Manchester tradicional.

3 pacientes, $4,4 \%$, histerectomía abdominal, 2 por fibromatosis y una no se conoce la causa.

La sintomatología de estas tres últimas pacientes se había presentado posterior a la histerectomía.

8 pacientes, $11,76 \%$ ten ían histerectomía vaginal previa, una paciente histerectomía abdominal sub-total postpartum.
Cuadro No. 2

ANTECEDENTES

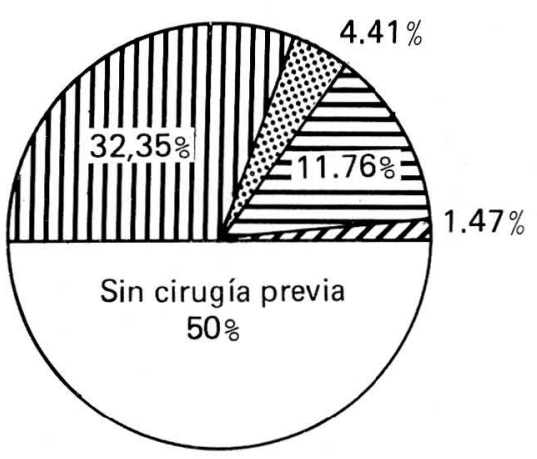

Convenciones:

DII Kelly - Kennedy y Manchester

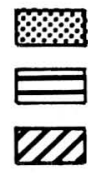

Histerectom ía Abdominal

Histerectomía Vaginal

Histerectom ía Abdominal

Subtotal

\section{Síntomas}

(Cuadro No. 3)

Incontinencia urinaria 68 casos (100\%).

Dolor hipogástrico 61 casos (89.7\%).

Infección arinaria a repetición 57 casos (83.82\%).

\section{Dispareunia 44 casos (64.7\%).}

Sensación de cuerpo extraño en la vagina 27 casos (39.7\%).

Otras pacientes se quejaban de prurito vaginal, salida de la matriz etc. Es de apreciar que el $100 \%$ consultó por incontinencia urinaria, que se encontraba asociada a sintomatología variada de tipo urológico y ginecológico. 
Cuadro No. 3

SINTOMAS

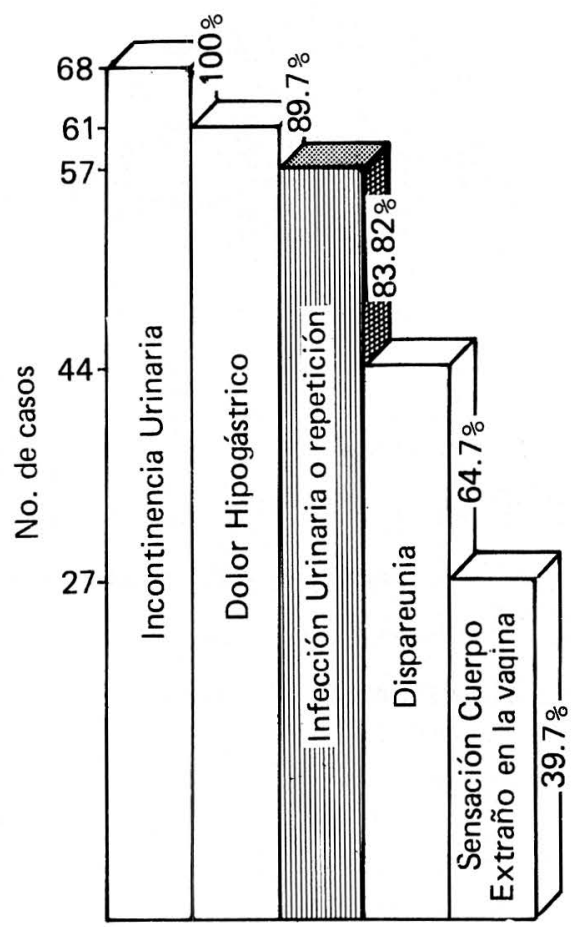

Hallazgos físicos

En el $100 \%$ de los casos se encontró cistocele en diversos grados, asociado a la incontinencia.

Se descartaron del estudio aquellas pacientes quienes consultaron por incontinencia y no se les halló cistocele clínicamente.

\section{Patología asociada}

(Cuadro No. 4)

Al examen ginecológico previo a la intervención se encontró:
Cuadro No. 4

PATOLOGIA ASOCIADA

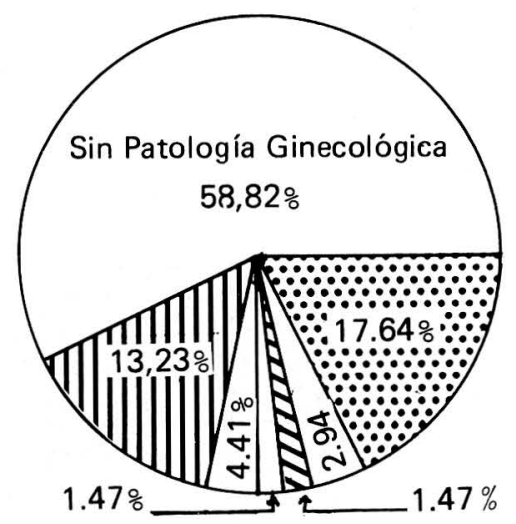

Convenciones:

$\because \because$ Prolapso Genital General

IIII Fibroma Uterino

$\square$ Hiperplasia Endometrial

$\square$ CA - Cervix

$\$$ Cuello Uterino Residual

$\square$ Prolapso Cupular Vaginal

28 pacientes (41.18\%) tenían patología ginecológica asociada a la incontinencia urinaria en el momento de la consulta.

Fibromatosis uterina 9 pacientes (13.23\%).

Prolapso genital general 12 pacientes (17.64\%).

Hiperplasia endometrial (Glánduloquística y adenomatosa) el $4.41 \% 3$ pacientes. 
Carcinoma in situ de cervix 1 caso (1.47\%).

Cuello uterino residual 1 caso (1.47\%).

Prolapso de cúpula vaginal 2 casos (2.94\%).

\section{Hallazgos cistoscópicos}

(Cuadro No. 5)

Al $100 \%$ de los pacientes se les practicó cistoscopia y se encontró: uretro trigonitis 58 casos (85.29\%), seudopapilomas en cuello vesical 35 casos (51.47\%), estenosis uretral 29 casos

\section{Cuadro No. 5}

\section{HALLAZGOS CISTOSCOPICOS}

No.

de

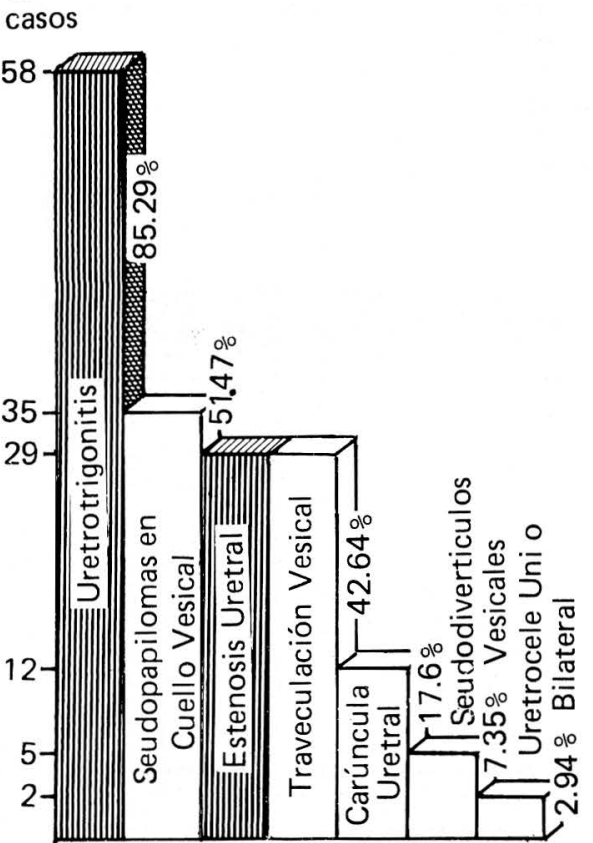

$(42.64 \%)$, traveculación vesical 29 casos (42.64응.

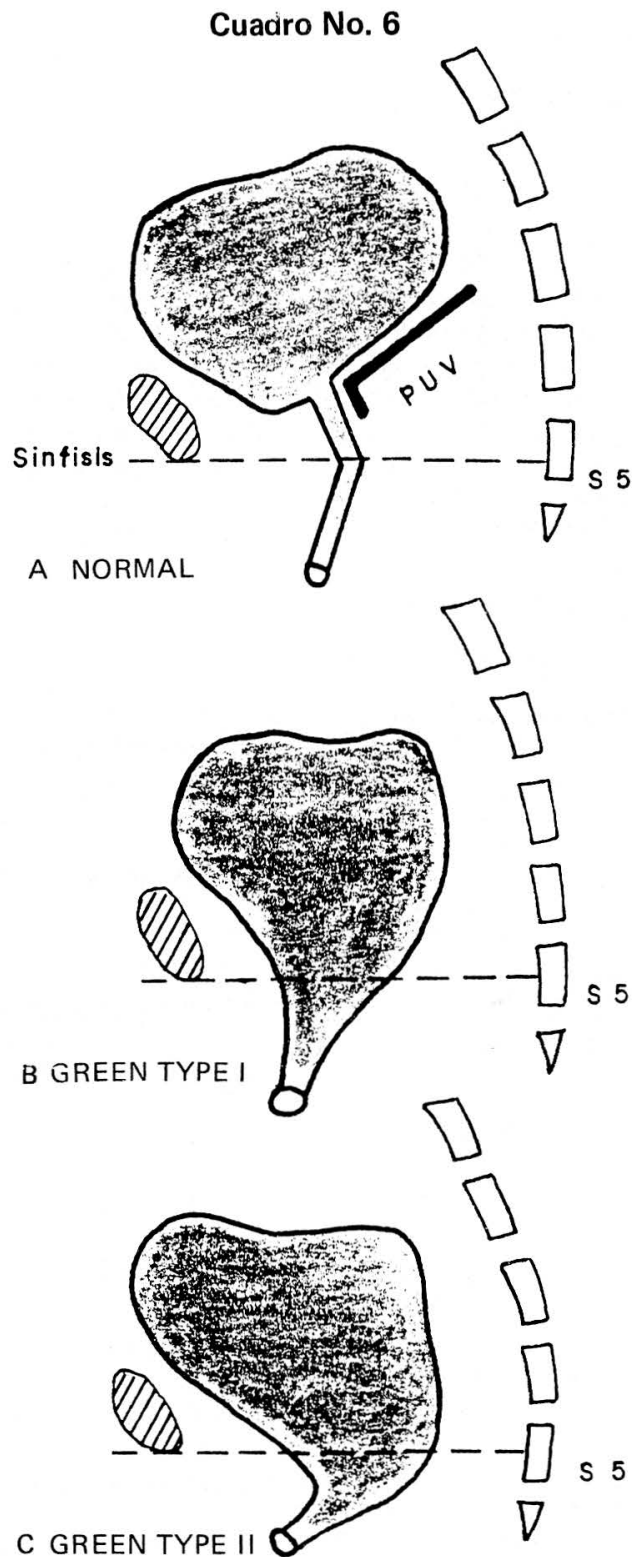


Carúncula uretral 12 casos (17.6\%), seudo-divertículos vesicales 5 casos (7.35\%), ureterocele uni o bilateral 2 casos $(2.94 \%)$.

\section{Tipificación bacteriana de la} infección urinaria

\section{( Cuadro No. 7)}

La infección urinaria se halló preponderantemente producida por gérmenes gran negativos, $y$ entre ellos predominó ampliamente el Escherichia Coli de diversos grupos, seguido por el Proteus, en sus diversas cepas; otros gérmenes se hicieron presentes pero su importancia es mínima. Esto igual que en todos los estudios de infección urinaria.

\section{RESULTADOS}

(Cuadro No. 8a.)

Se controlaron las 68 pacientes, o sea el $100 \%$ a los 30 días, observándose:
Resultado bueno: Incontinencia negativa, urocultivo negativo, 53 casos (77.94\%); aceptable: Incontinencia negativa, urocultivo positivo, 9 casos (13.24\%); malo Incontinencia positiva, urocultivo positivo, 6 casos (8.82\%).

\section{Control a 6 meses}

(Cuadro No. 8)

Resultado bueno, 27 casos (69.23); aceptable, 9 casos (23.07\%); malo, 3 casos (7.69\%).

Se llama resultado bueno el de aquellas pacientes a quienes se les negativizó el urocultivo y no se volvió a presentar incontinencia urinaria, bajo ninguna circunstancia.

Resultado aceptable el de las pacientes que teniendo urocultivos negativos o positivos se les presentaba la incontinencia urinaria solamente con grandes esfuerzos, como estornudos o accesos de tos.

\section{Cuadro No. 7}

\section{INFECCION URINARIA TIPIFICACION BACTERIANA}

E. Coli

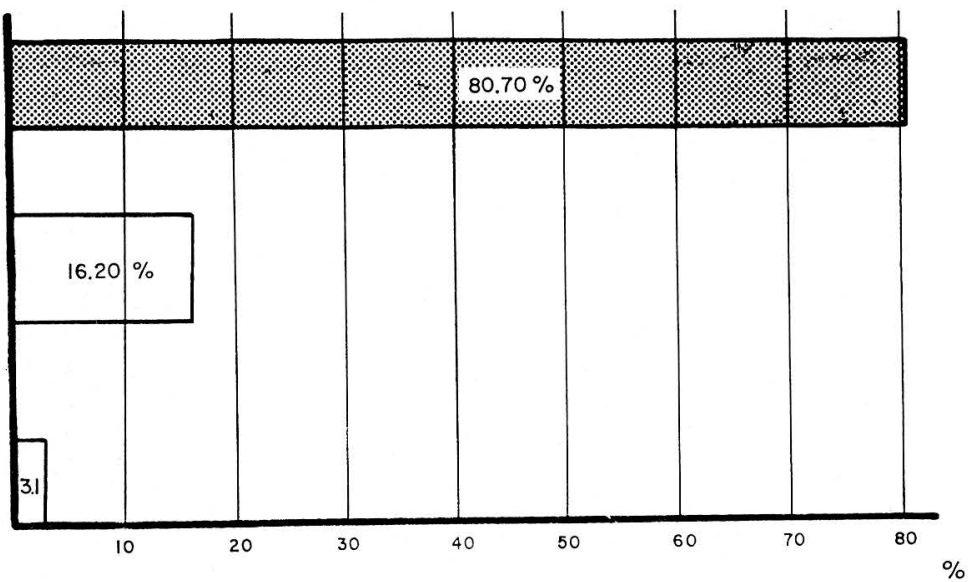


Cuadro No. 8 a.

Control a 6 meses

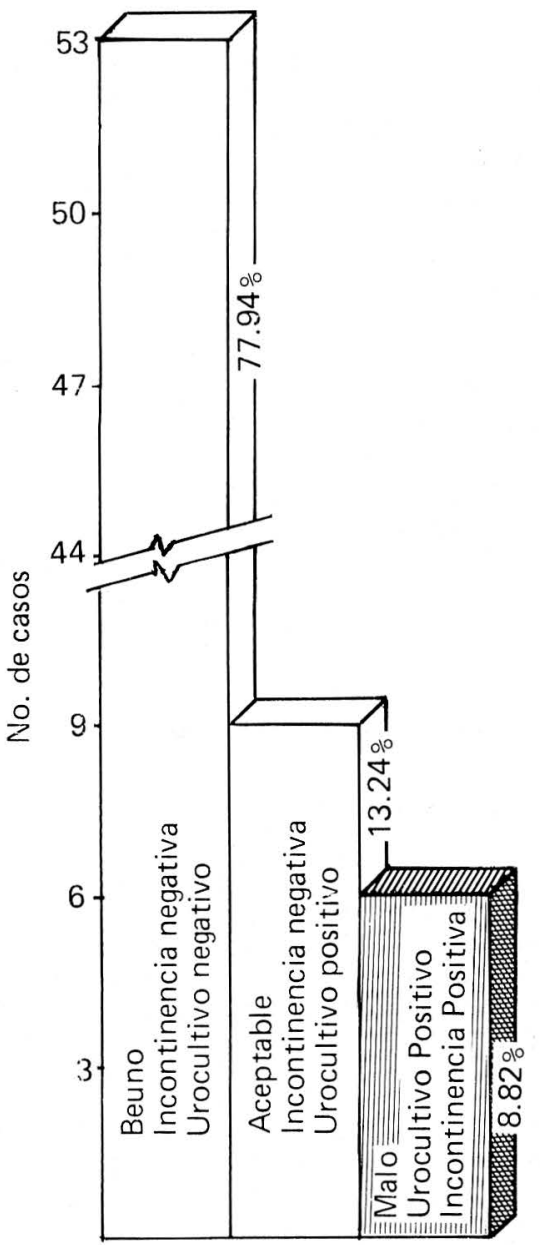

Cuadro No. 8

CORRECCION INCONTINENCIA URINARIA Control a 30 días

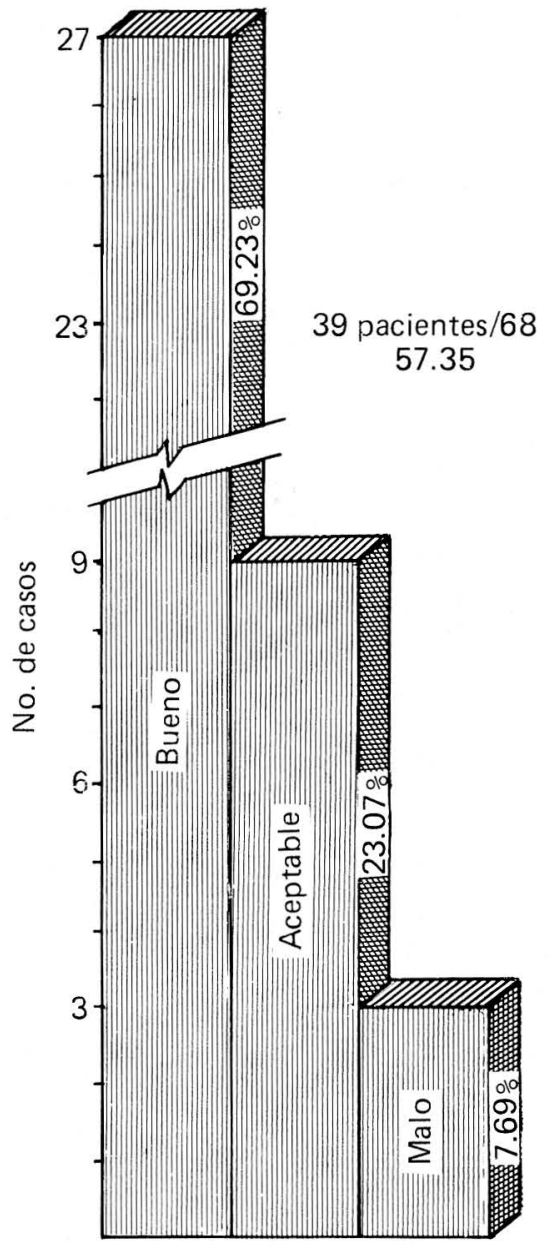

Resultado malo: el de las pacientes a quienes se les mantuvo el urocultivo positivo y la incontinencia urinaria se les presentaba por aumentos leves de la presión intra-abdominal.
También debemos anotar que a los 6 meses sólo se logró el control de 39 pacientes, que corresponden al 57.35\% de todos los casos intervenidos. Es de suponer que algunas de las pacientes 
que no regresaron a control a los 6 meses, estaban en la categoría de casos buenos o malos, que son los que habitualmente abandonan a su médico tratante.

\section{Cuadro No. 9}

\section{RELACION INCONTINENCIA-INFECCION URINARIA}

Control a 6 meses

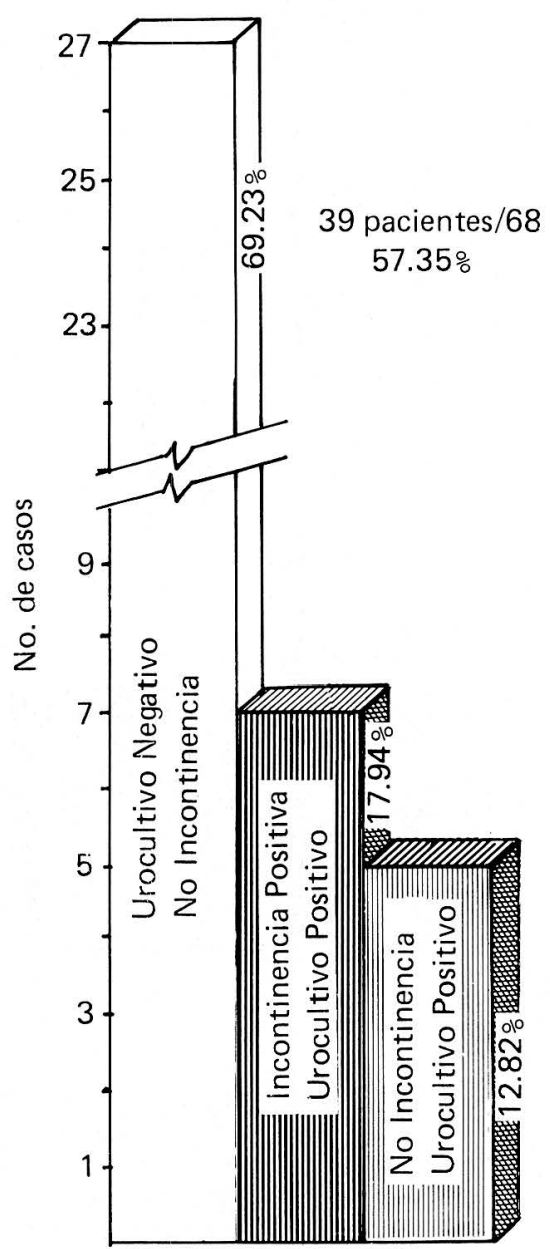

\section{Relación Incontinencia-Infección Urinaria control a 6 meses}

\section{Cúadro Nóo. 9}

Se halló que el 69.23\%, 27 pacientes, tenían incontinencia negativa y urocultivo negativo, es decir, un resultado bueno.

En el 17.94\%, 7 pacientes, se halló incontinencia positiva $\mathrm{y}$ urocultivo positivo.

$Y$ en $12.82 \%, 5$ pacientes, había urocultivo positivo, pero no existía incontinencia.

A todas las pacientes con urocultivo positivo se les trató específicamente, de acuerdo a los antibiogramas, esperando que al mejorar la infección urinaria, mejoraría la continencia.

\section{Incontinencia Urinaria Control a 1 año}

(Cuadro No. 10)

Se controlaron 16 pacientes sobre 68 , $23.52 \%$ del total. Se hallaron 11 pacientes, $16.17 \%$, con resultado bueno; esto sobre el gran total de 68 pacientes; 4 casos, $5.88 \%$, aceptable; 1 caso, $1.47 \%$, malo.

Aquí nuevamente hacemos la consideración sobre el gran total, ya que menos la cuarta parte de todas las pacientes regresaron al control a un año; esta baja obedece a diversas causas: la más importante el hecho de ser muchas de ellas pacientes protegidas por una entidad de Seguridad Social, y no obtuvieron o no solicitaron su consulta-control tardía, que en nuestro medio presupone un trámite de carácter administrativo, que para muchas personas es demasiado "engorroso". 
Cuadro No. 10

INCONTINENCIA URINARIA

Control a 1 año

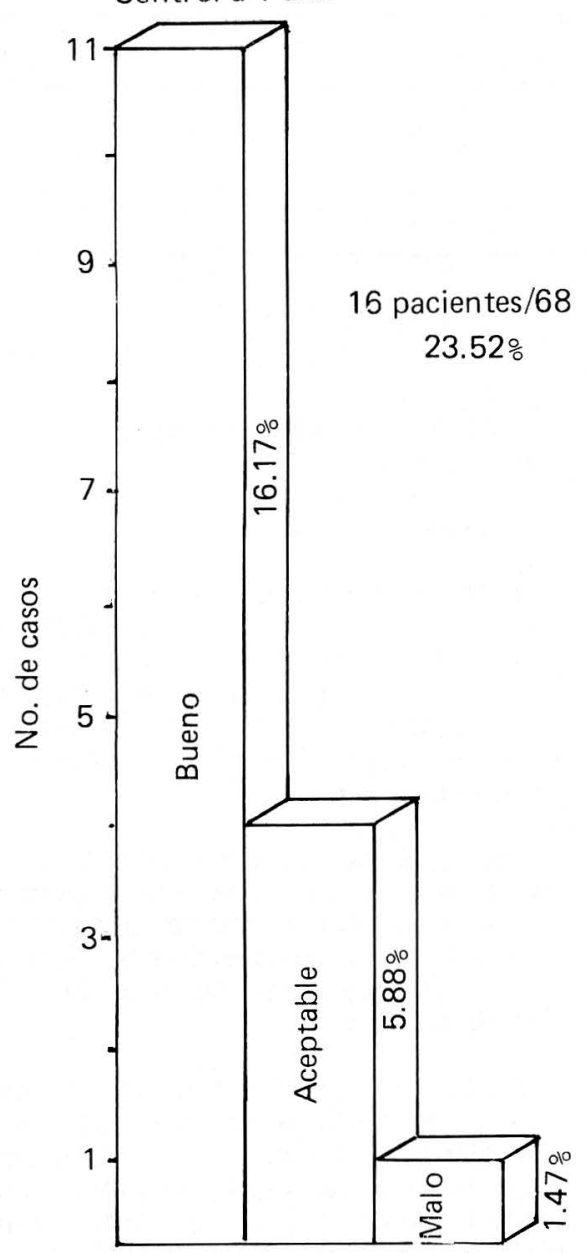

Relación Incontinencia-Infección Urinaria a un año

(Cuadro No. 11)

Resultado bueno: No incontinencia, urocultivo negativo, 11 casos (16.17\%);
Cuadro No. 11

RELACION INCONTINENCIA-INFECCION URINARIA

Control a 1 año

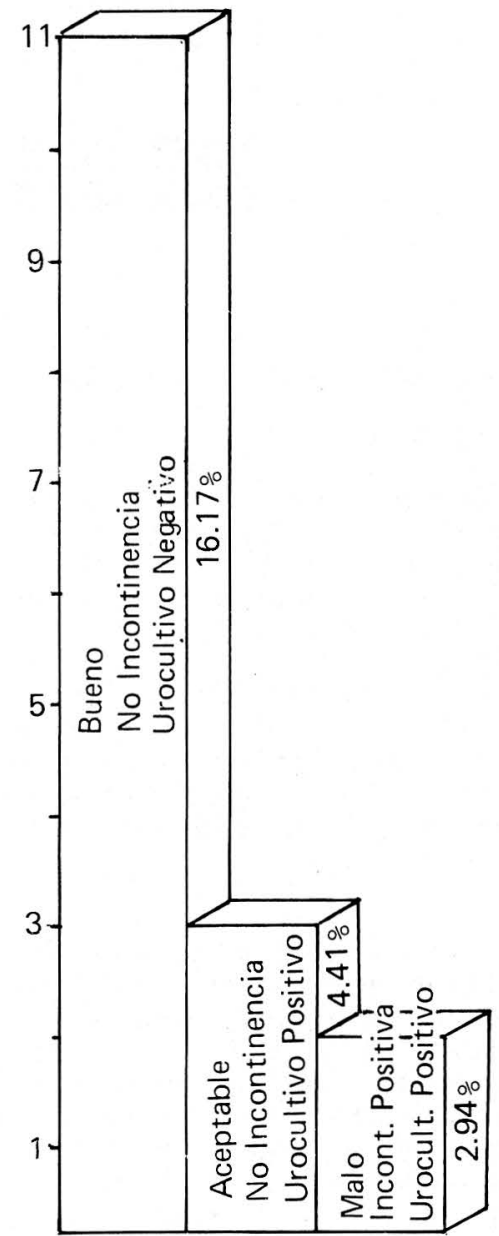

resultado aceptable: No incontinencia, urocultivo positivo, 3 casos $(4.41 \%)$; resultado malo: Incontinencia positiva, urocultivo positivo, 2 casos (2.94\%). 
Cuadro No. 12

COMPLICACIONES TRANS-OPERATORIAS

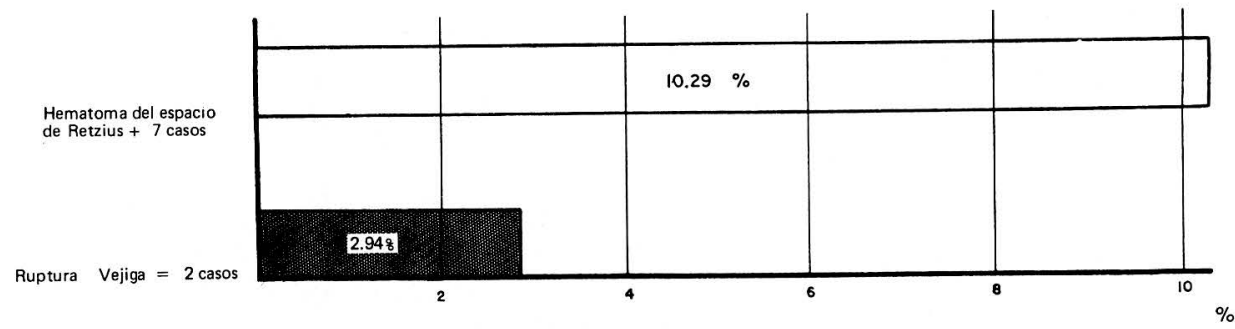

\section{Complicaciones Transoperatorias}

(Cuadro No. 12)

Se presentaron 7 casos de hematomas del espacio de Retzius, que corresponden a un 10.29\%; es de anotar que la gran mayoría se presentaron en nuestros primeros casos, cuando aún no dominábamos la técnica, a pesar de estar familiarizados con la disección de este espacio; en las intervenciones de próstata por vía suprapúbica, dicha complicación no ofrece problemas en su resolución, no siendo necesario recurrir a técnicas especiales.

Ruptura de vejiga: 2 casos (2.94\%). Se presentó este tipo de complicación en pacientes que previamente habían sido intervenidas y la disección digital del espacio se hizo muy laboriosa; en una de ellas que presentaba una obesidad exagerada, se produjo una disección de las fibras del detrusor, ocasionando la ruptura. Ambos casos se solucionaron sin complicaciones, mediante la sutura simple de la vejiga con cat-gut 000 , puntos no perforantes en dos planos.

En estas pacientes se dejó drenaje a permanencia de vejiga por una semana.

\section{Complicacicnes transoperatorias inmediatas}

(Cuadro No. 13)

Infección de herida quirúrgica: 4 casos que corresponden a un 5.88\%; el análisis de esta cifra es de valor relativo, pues en ésta los factores de influencia son múltiples y de muy difícil análisis en un estudio no encaminado a este tipo de complicación.

lleo paralítico: 3 casos (4.41\%) valor apenas normal en una serie de pacientes, donde se practica cirugía transperitoneal. Se maneja de acuerdo a las técnicas y normas habituales en este tipo de complicaciones.

Hemorragia post-operatoria: 1 caso $(1.47 \%)$ se trató de un caso donde la disección del espacio de Retzius fue especialmente laboriosa, se presentó la hemorragia en el post-operatorio inmediato, que se trató mediante transfusión. No fue necesario intervenir.

\section{Complicaciones Post-operatorias tardías}

Podemos considerar que la serie ofrece complicaciones propias al tipo de intervención practicada. Un caso de 
Cuaciro No. 13

\section{COMPLICACIONES POST-OPERATORIAS}

INMEDIATAS

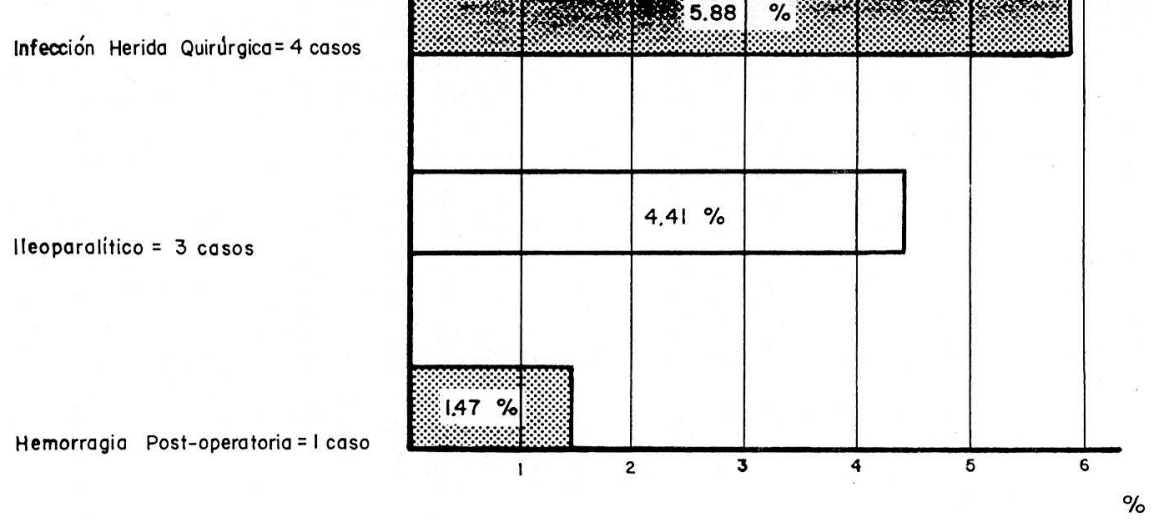

Cuadro No. 14

COMPLICACIONES POST-OPERATORIAS TARDIAS

Osteitis Pubis $=1 \cos 0$

Hernia Post-Incisional $=1$ caso

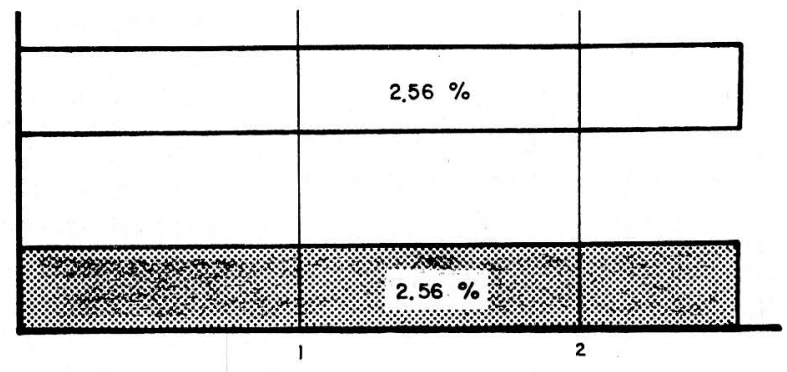

$\%$

osteitis del pubis, que se manifestó por los signos radiográficos de "panal de abejas" y se trató mediante la ampliación de calor, relajantes musculares y analgésicos, con la evolución tórpida propia de esta complicación obteniendo la remisión de los síntomas 2 meses después de la intervención. 
Hernia post-incisional: 1 caso (2.56\%).

\section{CONCLUSIONES}

Se han tomado 68 casos de incontinencia urinaria, $50 \%$ de los cuales presentaban recidiva de su problema a pesar de habérseles sometido a procedimientos quirúrgicos previos.

La técnica permite la corrección del ángulo uretro vesical posterior, usando la vagina para crear una banda de soporte, fijando ésta al ligamento de Cooper.

La corrección de la incontinencia urinaria y el problema ginecológico por una sola vía ofrece menor morbilidad, mayor facilidad en la técnica operatoria, y en general, un mejor pronóstico que en las técnicas tradicionales.

Consideramos que esta técnica operatoria ofrece al cirujano la oportunidad de hacer una mejor, más segura y senciIla toma de los puntos de fijación de la fascia vesico vaginal, lo cual conlleva a un mejor resultado transoperatorio, disminuyendo la tensión quirúrgica $y$ las complicaciones relacionadas a lesión iatrógena del aparato urogenital.

Como queda expuesto, en este informe preliminar de un procedimiento que obviamente debe ser sometido a las pruebas de depuración propias de una técnica quirúrgica, sufriendo el tamiz purificador del tiempo y el análisis real de aquellos que lo lleven a la práctica.

\section{MODIFIED CYSTOPEXIA}

\section{CONCLUSIONS}

This article analyzes the study of 68 cases of urinary incontinence, $50 \%$ of which relapsed despite undergoing previous surgical procedures.

The technique allows for correction of the posterior uretro-vesical angle, using the vagina to create a support band fixing it to the Cooper ligament.

Correction of urinary incontinence and the gynecological problem through one way only presents less morbidity, greater simplicity of operating technique and, in general, a better prognosis than with traditional techniques.

\section{BIBLIOGRAFIA}

1. BURCH. J.E. Uretrovaginal fixation to Cooper's ligament for the correction of stress incontinence, cystocele and prolapse. Am. J. Obstet. Gynecol. 81:281. 1961.
Authors consider that this operating technique provides the surgeon with the possibility of better, safer and more simple fixation points of vesicol-vaginal fascia, which implies better trans-operatory results, and diminishes surgical tension and complications related to iatrogenic damage of the urogenital apparatus.

As described in this preliminary report, this is a procedure that must be furtherly screened and tested as any other new surgical technique throughout time and day to day practice.

2. BURCH J.C. Cooper's ligament urethrovesical suspension for stress incontinence, Nine years experience, results, complications, technique, Am. J. Obstet. Gynecol 100: 764. 1968. 
3. GREEN. T.H. Jr. Development of a plan for the diagnosis and treatment of urinary stress incontinence. A M. J. Obstet. Gynecol. 83: 632. 1962.

4. HODGKINSON CP. Urinary stress incontinence. A program of preoperative investigation. Clin. Obstet. Gynecol. 6: 154. 1963.

5. HODGKINSON CP. Relationships of the female urethra and bladder in urinary stress incontinence Am. J. Obstet. Gynecol. 65: 560. 1953.

6. JEFFCOATE T.N.A., and ROBERTS, $H$. Stress incontinence of urine. J. Obstet. Gynaecol. Br. Commonw; 59. 685. 1952.

7. JEFFCOATE T.N.A. The principles governing the treatment of stress incontinence of urine in the female, $\mathrm{Br}$. J. Urol. 37: 633. 1965.

8. JEFFCOATE T.N.A. and ROBERTS H. Effects of urethrocystopexy for stress incontinence. Surg. Gynecol. Obstet. 98: 743. 1954.

9. KURSH E.O. WANSTEIN M. PERSKY L. The Pereyra procedure in urinary stress incontinence. J. Urclo. 108; 591, 1972.

10. MARSHALL V.F. Suprapubic vesicou rethral suspension for stress incontinence.
Gambell's Urology 4 Th edition Vol III 2294 W.B. Saunders. Phil. 1979.

11. MARSHALL V.F. SEGAUL R.M. Experiences with suprapubic vesicourethral suspension after previous failures to correct stress incontinence in women. J. Urol. 100; 647. 1968.

12. MARSHALL V.F. MARCHETTI. A.A. KRANTZ K.E. The correction of stress incontinence by simple vesicourethral suspension. Burg. Gynecol. Obstet. 88; 590. 1949.

13. PEREYRA A.J. A simplified surgical procedure for correction of stress incotinence in women West. J. Surg. 67: 223.1959.

14. STAMEY THOMAS A. Urinary incontinence in the female. Campbell's Urology 4th edition Vol III Chp 75 2272. W.B. Saunders CO. 1979. Philadelphia. 1979.

15. STAMEY T.A. Endoscopic suspension of the vesical neck for urinary incontinence. Surg. Gynecol. Obstet. 136: 547. 1973.

16. THANAGO E.A.: Simplified cystography in stress urinary incontinence $\mathrm{Br}$. J. Urol., 46: 295. 1974.

17. T. LINDE, R.W. MATTINGLY R.F. Relaxed vaginal outlet, rectocele and enterocele. In Operative Ginecology. 4th Ed. p 624. Phil. J.B. Lipincott Co. 1970. 